\title{
EPR Dating of Hydroxyapatite from Fossil Bones. Transient Effects after $\gamma$ and UV Irradiation
}

\author{
M. WenckA ${ }^{a, *}$, S.K. HoffmanN ${ }^{a}$ AND H. Hercman ${ }^{b}$ \\ ${ }^{a}$ Institute of Molecular Physics, Polish Academy of Sciences \\ Smoluchowskiego 17, 60-179 Poznań, Poland \\ ${ }^{b}$ Institute of Geological Sciences, Polish Academy of Sciences \\ Twarda 51, 00-818 Warszawa, Poland
}

\begin{abstract}
ESR measurements at room temperature have been performed on fossil bones of cave bear (from Magurska Cave of the Tatra Mountains) and mammoth (from Dniestr River). Various paramagnetic centres related to defects in hydroxyapatite were identified. The ESR spectrum is dominated by $\mathrm{Mn}^{2+}$ lines and free radical centres $\mathrm{CO}_{2}^{-}$and $\mathrm{CO}_{3}^{-}$. Suitability of various EPR lines for the dating was evaluated. The spectra are affected by $\gamma$-ray and UV irradiation which produce new relatively unstable (transient) radical centres. From their decay curves we determined the delay period (waiting time) between irradiation and ESR dating measurements. For $\gamma$-rays the period is about 15 days and depends on the chosen ESR line. UV generates mainly surface $\mathrm{O}^{-}$centres which decay practically after a few days. The ESR dating of the bear bones using an additive dose method given the age $t=21.7$ kiloyears (ky), whereas from radionuclide $\mathrm{Th} / \mathrm{U}$ method $t=34 \mathrm{ky}$ and from radiocarbon ${ }^{14} \mathrm{C}$ method $t>33.1 \mathrm{ky}$.
\end{abstract}

PACS numbers: 76.30.- $-\mathrm{v}, 65.90 .+\mathrm{i}$

\section{Introduction}

Various physical techniques, spanning various time intervals, are used for dating in archaeology, geology, and dendrochronology [1-3]. Some of the methods detect long lived defects produced by natural radiation (uranium, thorium, potassium) and trapped in minerals or biomaterials. Among them the non-destructive method is electron spin resonance (ESR) which detects paramagnetic defects like

*corresponding author; e-mail: mwencka@ifmpan.poznan.pl 
trapped free radicals or hole centres allowing dating in time interval from 5000 years to about 1 million years. Other dating techniques like radiocarbon or $\mathrm{K} / \mathrm{Ar}$ dating are not sensitive enough in this relatively short time interval. ESR is used for dating minerals and rocks containing paramagnetic defects related to $\mathrm{SiO}_{2}$, cave deposits with defects in calcite, biocarbonate defects in fossils and sediments as shells, corals or molluscs, apatite related defects in bones, tooth enamels and dentine [1, 4]. Human and animals bones and teeth are very suitable for dating [1, 5-7], for an estimation of absorbed dose of irradiation (like for Hiroshima A-bomb victims) $[1,8,9]$ and for detection of irradiated foodstuffs containing bones or fish-bones [10].

Bones and teeth consist of inorganic compound hydroxyapatite $\mathrm{Ca}_{10}\left(\mathrm{PO}_{4}\right)_{6}(\mathrm{OH})_{2}$ in which paramagnetic defects can be accumulated and used as signals for dating. Synthetic and sintered apatites irradiated with $\gamma$-rays and X-rays or doped with paramagnetic impurities have been studied by ESR $[11,12]$ and by ENDOR techniques [13, 14] giving a background for interpretation of ESR spectra of natural materials. Several paramagnetic species located at hydroxy or phosphate sites have been identified in synthetic hydroxyapatite : oxygen radical $\mathrm{O}^{-}$, trapped atomic hydrogen, and hole trapped on $\mathrm{OH}^{-}$and $\mathrm{PO}_{4}^{2-}[12]$. Moreover, carbonate radicals $\mathrm{CO}_{2}^{-}, \mathrm{CO}_{3}^{-}$, and $\mathrm{CO}_{3}^{3-}$ are often observed as a result of contamination by calcium carbonate [14]. In bones and teeth the EPR spectra are usually dominated by $\mathrm{CO}_{2}^{-}$signals $[1,15]$ but also additional organic radicals (alanine-like) signals [16] and colour centres signals [17] appears. In burnt bones the coal-type $C^{\bullet}$ radicals were identified [18].

An additive dose method of dating by ESR technique is based on measurements of a dependence of the intensity or amplitude of selected ESR signals on increasing dose of radiation. Thus the first step in the procedure is an interpretation of the ESR spectrum with identification of paramagnetic centres and selection suitable lines for dating. In this paper we identify paramagnetic defects in bones of a cave bear and fossil mammoth.

Identification of the centres which were accumulated during geological periods and its separation from another centres giving ESR signals makes still difficulties. ESR spectra as a rule consist of a lot of overlapping lines which can be resolved using computer methods of the resolution enhancement [19]. Moreover, the spectra are affected by sample preparation procedures as chemical treatment (etching), annealing, crushing, grinding and depend on the grain size (a grinding very often produces a new ESR signal at $g=2.001)$ [20]. Some of these effects, especially temperature effects, for calcite samples of speleothems we have described recently [21]. Also storage conditions can influence ESR spectra by oxidation of organic impurities and by paramagnetic centres induced by UV-components of the sun light.

Additional dose method results can be disturbed also by an apparent fading of the dating signal after $\gamma$-irradiation. The four fading signals were identified 
in [17] with the decay period order of two weeks. Among them was the coal-type $\mathrm{C}^{\bullet}$-radical at $g=2.0086$ and organic radical signal at $g=2.0046$.

In the present paper we describe and discuss the $\gamma$-ray and UV-irradiation transient radical signals. We show their influence on various ESR lines of initial spectra and we suggest the lines preferable for dating. We indicate also the delay periods (waiting times) between irradiation and ESR measurements necessary for decay the transient radicals.

\section{Experimental}

Samples of fossil bones of cave bear were extracted from the Magurska Cave (The Western Tatra Mountains, Poland) and mammoth bone from the slope of the Dniestr River (near Halicz, Ukraina). The soft and porous layers of bones were removed. The next stages of procedure of the sample preparation were as described previously [21]. Samples of the cave bear bones used for EPR dating were $\gamma$-irradiated with ${ }^{60} \mathrm{Co}$ source with doses up to $300 \mathrm{~Gy}$. Additionally a process of recombination of radicals generated by the dose of 150 Gy was studied for 98 days.

The sample of mammoth bone was divided into two parts. The first part was irradiated by natural sunlight (in the summer time) for 161 days. The second part of the bone was UV irradiated using an EMITA VP-60 lamp at $254 \mathrm{~nm}$, $315 \mathrm{~nm}$, and $366 \mathrm{~nm}(410 \mathrm{~W})$ for 6.5 hours and then for 161 days the process of recombination of radicals was detected by ESR.

ESR measurements were performed on a Radiopan SE/X-2547 working at $\mathrm{X}$-band, with $100 \mathrm{kHz}$ magnetic modulation and a home-made high temperature ESR cavity of $\mathrm{TE}_{102}$ type.

\section{Results and discussion}

ESR spectra of the bear and mammoth bones are very similar, with better resolution for the latter because of narrower lines (lower radical concentration). The mammoth bone ESR spectrum is shown in Fig. 1 (upper spectrum). Central part of the spectrum, located between two hyperfine lines of $\mathrm{Mn}^{2+}$ impurities, shows peaks from "axial" $\mathrm{CO}_{2}^{-}$and "isotropic" $\mathrm{CO}_{3}^{-}(g=2.0118)$ radicals, which are characteristic of bones and enamels samples [1]. After $\gamma$-ray irradiation the total intensity of the ESR spectrum increases and the spectrum is dominated by $\mathrm{CO}_{2}^{-}$signals at $g_{\|}=1.9970$ and $g_{\perp}=2.0020$. The $g_{\perp}$-line is very intensive because of overlapping with lines from surface $\mathrm{O}^{-}$centres and colour centres $\mathrm{F}$.

These new centres and other mentioned in the Introduction, are generated by the $\gamma$-rays and are unstable. They overlap with main lines of the initial spectrum and their decay is observed as a decrease of the spectrum intensity in time. The decay curves of apparent amplitude of the $\mathrm{CO}_{2}^{-}$and $\mathrm{CO}_{3}^{-}$(overlapped with $\mathrm{PO}_{3}^{-}$) 


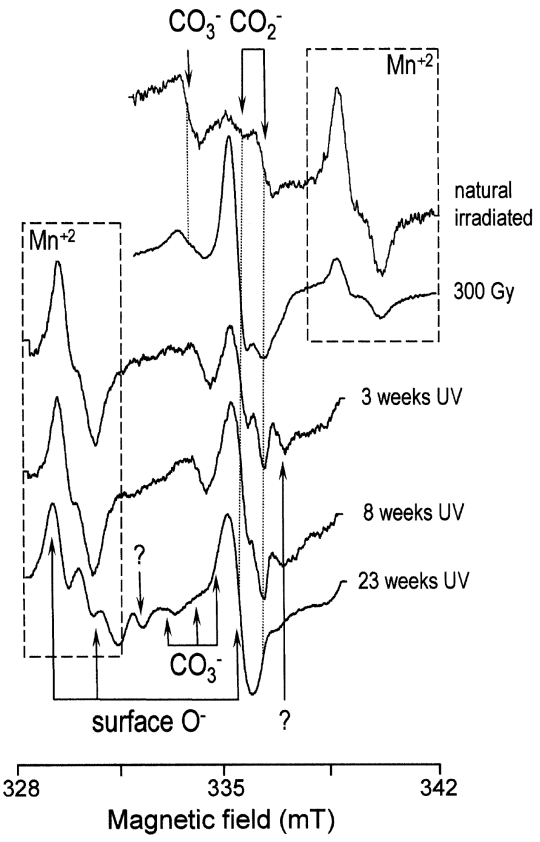

Fig. 1. ESR spectra of natural, $\gamma$-irradiated and long-time sunlight irradiated fossil mammoth bones. The central part of the spectra is dominated by $\mathrm{CO}_{2}^{-}$and $\mathrm{CO}_{3}^{-}$ radical lines surrounded by two hyperfine lines $\left(m_{I}= \pm 1 / 2\right)$ of $\mathrm{Mn}^{2+}$ impurity ions.

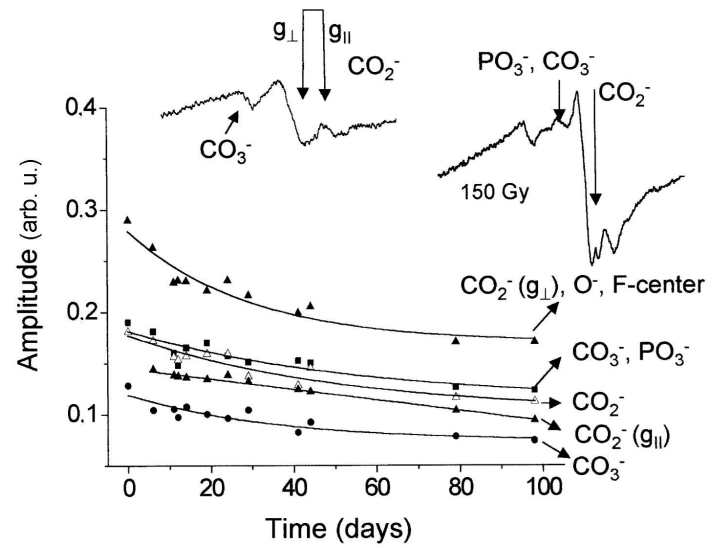

Fig. 2. Decay curves of the ESR line amplitudes for $\mathrm{CO}_{2}^{-}$and $\mathrm{CO}_{3}^{-}$radicals after $\gamma$-ray irradiation with a dose of $300 \mathrm{~Gy}$. The spectra of the cave bear bones were recorded: left — before $\gamma$-irradiation; right - after 150 Gy $\gamma$-irradiation.

signals are shown in Fig. 2. The decay is rather slow and shows that the spectrum is dominated by accumulated long-lived radicals, which can be used for dating. Only $g_{\|}$-line of $\mathrm{CO}_{2}^{-}$centre decays relatively faster suggesting that it is overlapped 
with lines of a few transient radicals. The decay behaviour indicates that dating with $\mathrm{CO}_{2}^{-}$and $\mathrm{CO}_{3}^{-}$signals can be safely performed after about 15 days waiting time after irradiation, and only for $g_{\perp}$-line of $\mathrm{CO}_{2}^{-}$(this line is commonly used for dating) the waiting time should be longer than 40 days.

For the samples which were exposed on the natural sun light at environmental conditions the carbonate radicals ESR lines grown linearly with exposure time with rate doubling the line amplitudes in about 10 weeks. Only $g_{\perp}$-line of $\mathrm{CO}_{2}^{-}$has a larger grow rate (about 1.5 times higher). The changes in the EPR spectrum of the mammoth bone may be detected in EPR spectra up to 23 weeks of exposure as shown in Fig. 1. The main effects is due to oxygen centre $\mathrm{O}^{-}\left(g_{z}=2.0023, g_{y}=\right.$ $\left.2.0330, g_{x}=2.0414\right)$. The $g_{z}$-line of this radical is superimposed on $g_{\perp}$-line of $\mathrm{CO}_{2}^{-}$and dominates in the spectrum for long-time exposures. Moreover, lines from unidentified centres appear for long times and are marked with question marks in Fig. 1.

Similar effects to the above were observed when samples were irradiated with the UV-lamp (up to $6.5 \mathrm{~h}$ ). After the irradiation a fast decay of the generated centres was observed with the decay rate much higher than for the transient radicals generated by $\gamma$-rays. The decay curves are shown in Fig. 3 for $\mathrm{CO}_{2}^{-}$and $\mathrm{CO}_{3}^{-}$ radicals. The decay time of the transient radicals is here of the order of a few days and this is the suggested waiting period prior to the dating, for samples stored at environmental conditions.

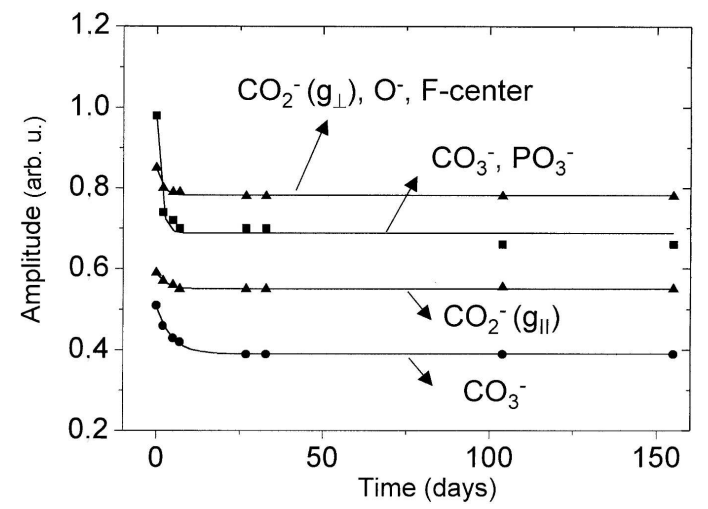

Fig. 3. Decay curves of ESR lines of the mammoth bones after irradiation with UV-lamp.

We have performed the dating procedure for the cave bear bones since the natural dose of radiation has been not assessed during excavation of the mammoth at Ukraine. The dating was performed using ESR lines of $\mathrm{CO}_{2}^{-}$. An influence of the additive $\gamma$-ray dose on the line amplitudes is shown in Fig. 4. The linear dependence of the amplitude on dose is a good approximation, but different intercepts with the dose-axis exist for the two extrapolated lines. The intercept 


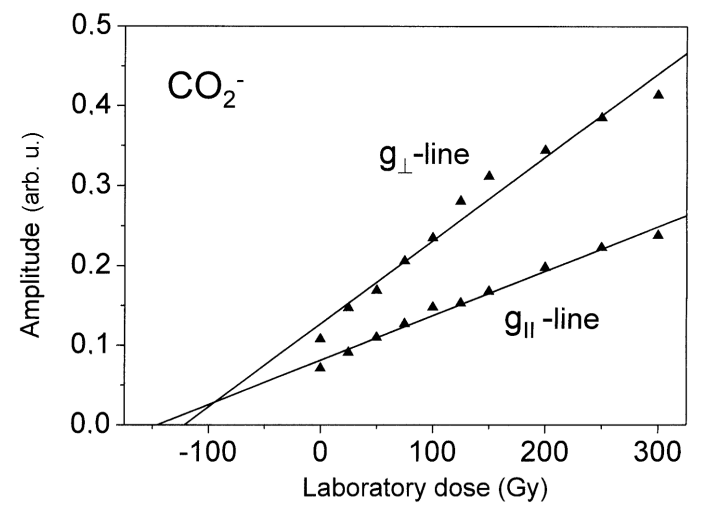

Fig. 4. The additive dose method lines representing changes in ESR line amplitudes for the $g_{\|}$-line and $g_{\perp}$-line of $\mathrm{CO}_{2}^{-}$radical. The total dose determined from the intercept of the straight line for $g_{\|}$is equal to $48 \mathrm{~Gy}$ (see discussion in the text).

determines the total dose $(T D)$ of the radiation absorbed by the sample during a geological period, and $T D$ determination is critical for accuracy of ESR dating.

Because the both considered lines have the same apparent origin, i.e. $\mathrm{CO}_{2}^{-}$ radical, thus its $T D$ s should be the same. However, the higher slope for the $g_{\perp}$-line suggests that except $\mathrm{CO}_{2}^{-}$another centres are generated by $\gamma$-rays $\left(\mathrm{O}^{-}\right.$, organic radicals, colour centres) with ESR lines at the same position (similar $g$-factors). Thus we observed so-called superlinearity for the $g_{\perp}$-line [22], which should be corrected to obtain pure effect for the carbonate radical. Instead of this we can use the $g_{\|}$-line of $\mathrm{CO}_{2}^{-}$centre which is not disturbed by additional contributions. Thus the total dose of radiation absorbed by the sample can be evaluated as $T D=148$ Gy.

Age of the sample is calculated as $t=T D / D$, where $D$ is an annual dose rate of the radiation. The annual dose rate has been determined [19] using methods described in [1] according to the uranium-disequilibrium model [23] as $6.8 \mathrm{mGy} / \mathrm{y}$ (external annual dose rate has been measured as $0.063 \mathrm{mGy} / \mathrm{y}$ ). This assessment gives a sample age $t=21.7 \pm 3.5$ kyears.

Independent determination of the sample age has been performed by "classical" radioisotopic ${ }^{230} \mathrm{Th} /{ }^{234} \mathrm{U}$ method and radiocarbon ${ }^{14} \mathrm{C}$ method. The determined age is $t=34 \pm 3$ kyears from ${ }^{230} \mathrm{Th} /{ }^{234} \mathrm{U} t>33.1$ kyears from ${ }^{14} \mathrm{C}$. Thus the cave bear age determined by the three different methods is the same order of magnitudes and can be taken as an average value $t=30$ kyears $( \pm 8)$.

The paper shows that a selection of an EPR line for dating and using appropriate waiting periods after irradiation can be crucial for the sample age determination. 


\section{Acknowledgment}

The study was financially supported by the Ministry of Scientific Research and Information Technology under grant 3 P04D 03022 and grant 6 P04D 05615. We are indebted to the Gliwice Radiocarbon Laboratory for the ${ }^{14} \mathrm{C}$ dating of the cave bear bones sample Magurska I/5.

\section{References}

[1] M. Ikeya, New Applications of Electron Spin Resonance - Dating, Dosimetry and Microscopy, World Sci., Singapore 1993.

[2] Chronometric Dating in Archaeology, Eds. R.E. Taylor, M.J. Aitken, Plenum, New York 1997.

[3] H.P. Schwarcz, Acc. Chem. Res. 35, 637 (2002).

[4] M. Ikeya, Adv. ESR Appl. 18, 321 (2002).

[5] M. Ikeya, T. Miki, Science 207, 977 (1980).

[6] A.D. Oduwole, K.D. Sales, Nucl. Tracks Radiat. Meas. 18, 213 (1991).

[7] K.D. Sales, A.D. Oduwole, G.V. Robins, S. Olsen, Nucl. Tracs 10, 845 (1985).

[8] S. Mascarenhas, A. Hasegawa, K. Takeshita, Bull. Am. Phys. Soc. 18, 579 (1973).

[9] Use of Electron Paramagnetic Resonance Dosimetry with Tooth Enamel for Retrospective Dose Assessment, IAEA, Vienna 2002.

[10] M.F. Desrosiers, Appl. Radiat. Isotop. 47, 1621 (1996).

[11] H. Ishii, M. Ikeya, Appl. Radiat. Isotop. 44, 95 (1993).

[12] D.M. Close, M. Mengeot, O.R. Gilliam, J. Chem. Phys. 74, 5497 (1981).

[13] P. Moens, F. Callens, S. Van Doorslaer, P. Matthys, Phys. Rev. B 53, 5190 (1996).

[14] J. Sadlo, P. Matthys, G. Vanhaelwym, F. Callens, J. Michalik, W. Stachowicz, J. Chem. Faraday Trans. 94, 3275 (1998).

[15] M. Mengeot, R.H. Bartram, O.R. Gilliam, Phys. Rev. B 11, 4110 (1975).

[16] M. Ikeya, Dating Methods of Pleistocene Deposits and their Problems, in Geoscience Canada, Ed. W. Rotter, Geol. Assoc. Canada, Edmonton 1985, p. 71.

[17] A.D. Oduwole, K.D. Sales, Quarter. Geochron. 13, 647 (1994).

[18] M. Ohta, M. Yasuda, H. Okamura, Rad. Protect. Dosim. 94, 385 (2001).

[19] M. Wencka, R. Krzyminiewski, Appl. Magn. Res. 26, 561 (2004).

[20] D.F. Regulla, A. Wieser, H.Y. Goksu, Nucl. Tracks 10, 825 (1985).

[21] M. Wencka, S.K. Hoffmann, R. Krzyminiewski, S. Mielcarek, Acta Phys. Pol. A (2005) in press.

[22] A.D. Oduwole, K.D. Sales, K.J. Dennison, Appl. Radiat. Isotop. 44, 261 (1993).

[23] T. Goslar, H. Hercman, Quart. Sci. Rev. 7, 423 (1998). 